\title{
3D Elevation Program Supports Broadband Internet Access
}

\section{Introduction}

Broadband, which is a high-speed, always-on internet connection, is critical to the modern economy and education, as well as to healthcare, public safety, government services, entertainment, and more. However, according to the Federal Communications Commission (FCC), millions of Americans in rural parts of the country currently lack access to broadband. Federal and State agencies have launched initiatives to enhance broadband access in rural America. High-resolution light detection and ranging (lidar) data can play a role in improving maps of broadband accessibility and helping to strengthen the broadband communications network. Lidar underpins signal propagation analyses to determine where broadband signals from communication towers can and cannot reach across the landscape and can be used to determine optimal siting of new towers and cell stations to increase broadband coverage.

The 3D Elevation Program (3DEP; see sidebar) is managed by the U.S. Geological Survey in partnership with Federal, State, Tribal, U.S. territorial, and local agencies to acquire consistent lidar coverage for the Nation. Lidar provides high-resolution, very accurate three-dimensional (3D) data representations of constructed and natural features on the Earth's surface. Bare-earth and digital surface models derived from lidar are used in viewshed analyses for signal propagation studies necessary to identify the optimum locations for cell tower and cell station networks. Viewshed analysis, also known as line-of-sight analysis, determines the visibility of objects or areas from different points of view, such as from the top of a transmission tower (fig. 1). Objects such as buildings, hills, and other features (referred to as clutter in the communications industry) can attenuate or block signals, which reduces the coverage area of a broadband signal transmitter.

\section{Rural Broadband Access}

Expanding broadband access to underserved rural communities will provide entry for internet applications that are becoming increasingly critical to the modern economy and education system. Not only will fifth-generation (5G) wireless cellular technology networks support cell phones and computer systems, but they will also support devices such as medical equipment, security cameras, traffic signals, robots for manufacturing or delivery, and other

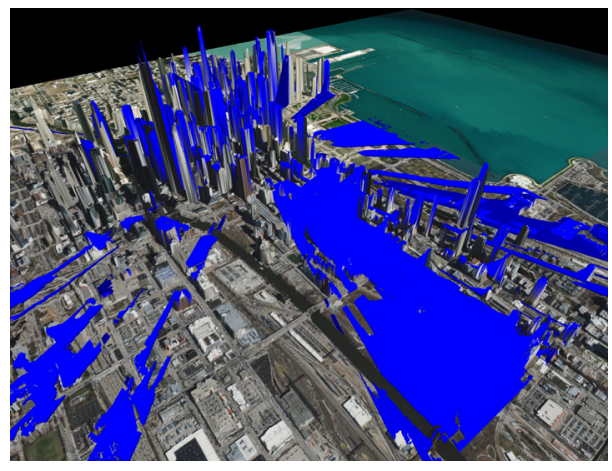

Figure 1. Simulated viewshed mapping from a transmitter located on top of a building in Chicago, Illinois, based on lidar data. Blue represents buildings and areas that are visible from the transmitter location. Graphic by Cindy Thatcher, U.S. Geological Survey.

elements of the "Internet of Things." For example, precision agriculture requires access to broadband from all corners of a farm field for the operation of highly advanced agricultural equipment. 5G networks rely more on the highband spectrum to achieve faster speeds over shorter distances but are also more susceptible to interference from clutter. Lidar can be used to determine the heights of nearby silos (often the highest location on a farm) where antennas could be located, and a viewshed analysis using lidar can help to identify where the signal can and cannot reach. This type of analysis could contribute to efforts to map the location of structures that require broadband access, referred to as the "fabric of broadband serviceable structures."

\section{Determining Broadband Coverage}

Cambium Networks, a wireless connectivity solutions provider that furnishes cloud-managed Wi-Fi and fixed wireless broadband, is using 3DEP lidar data to map wireless connectivity for customers and their communities. In areas where high-resolution lidar data are available, Cambium Networks uses the data to model signal propagation to more accurately predict radio frequency coverage (fig. 2). This information accelerates installation efficiency of fixed wireless services by enabling the installer to go to the precise location to maximize wireless connectivity and avoid installations where service is not possible.

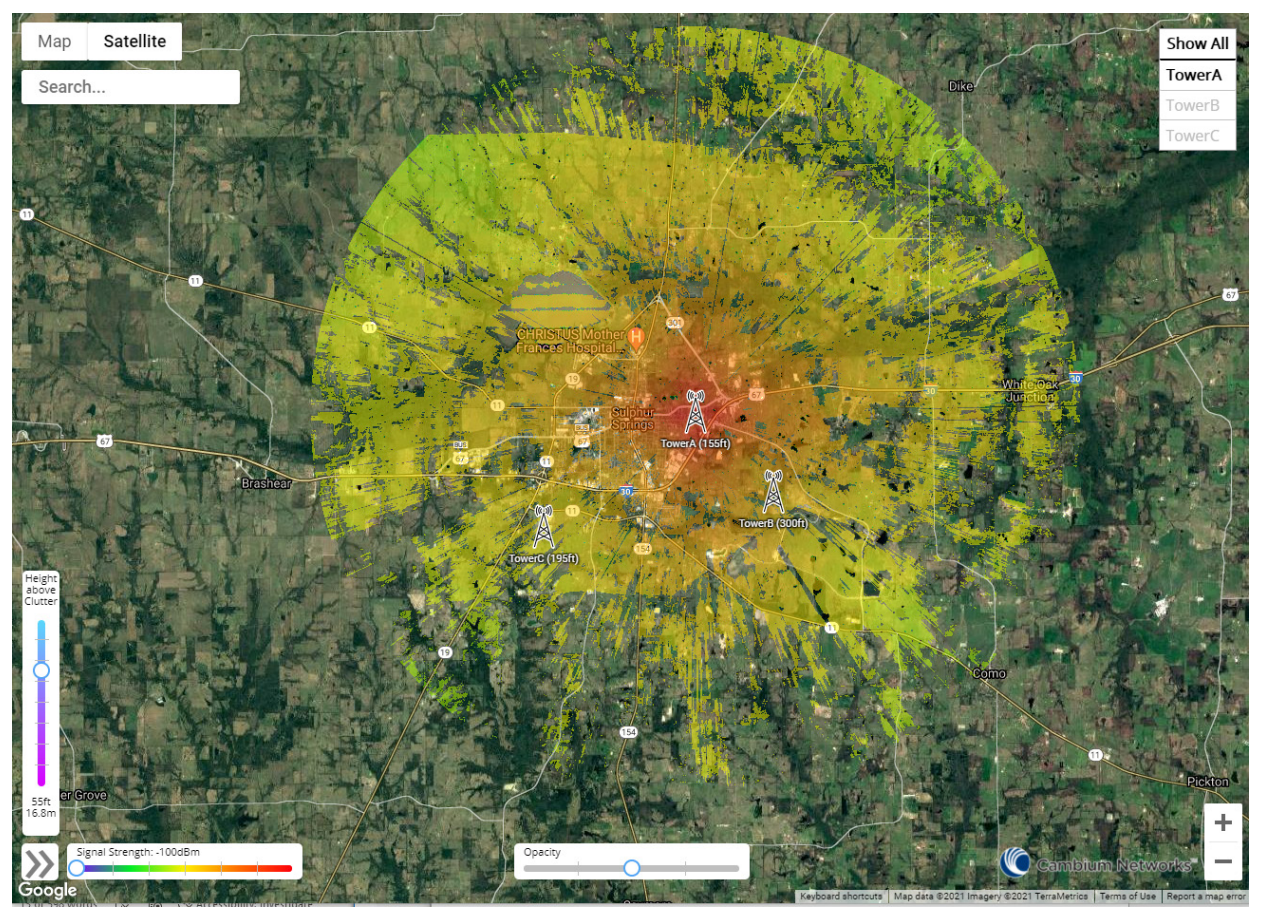

Figure 2. Screenshot of an interactive model of wireless broadband coverage developed by Cambium Networks. The model uses 3D information on obstructions such as trees and buildings, combined with signal strength and tower height, to predict wireless broadband coverage. The simulated tower height is 55 feet above the clutter, the opacity of the signal strength coverage is set to 50 percent, and the signal strength is expressed as decibel-milliwatts $(\mathrm{dBm})$. Model area is Sulphur Springs, Texas, and vicinity. Graphic courtesy of Cambium Networks; used with permission. 


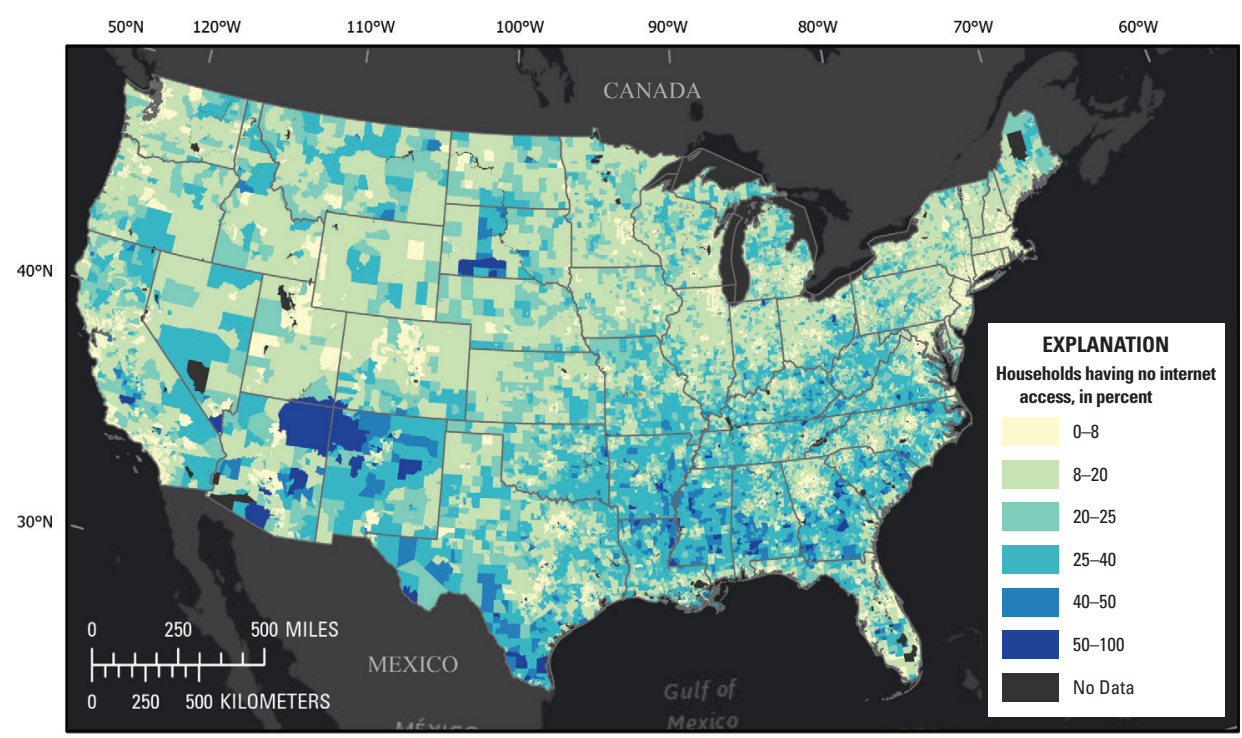

Figure 3. Percentage of households within a census tract that do not have access to the internet in the United States. The map displays National Telecommunications and Information Administration data derived from the U.S. Census American Community Survey taken during the period 2015-2019. Source: Federal Communications Commission.
The National Telecommunications and Information Administration indicators of broadband need provides information on the percentage of households reporting no internet access by census tract (fig. 3). Lidar data (fig. 4) could be used as an input to models of signal propagation from existing communications infrastructure to improve the detail and accuracy of broadband coverage maps. More detailed broadband availability data could be used to inform decision making on broadband policy and investments to help bridge the digital divide between rural and urban America.
Figure 4. A lidar pointcloud image in Olympia, Washington, capturing trees, buildings, and other objects that can attenuate or block broadband signals. Colors represent elevation; while treetops range from yellow to red. Image courtesy of Cindy Thatcher, U.S. Geological Survey. the riverbed is dark blue,

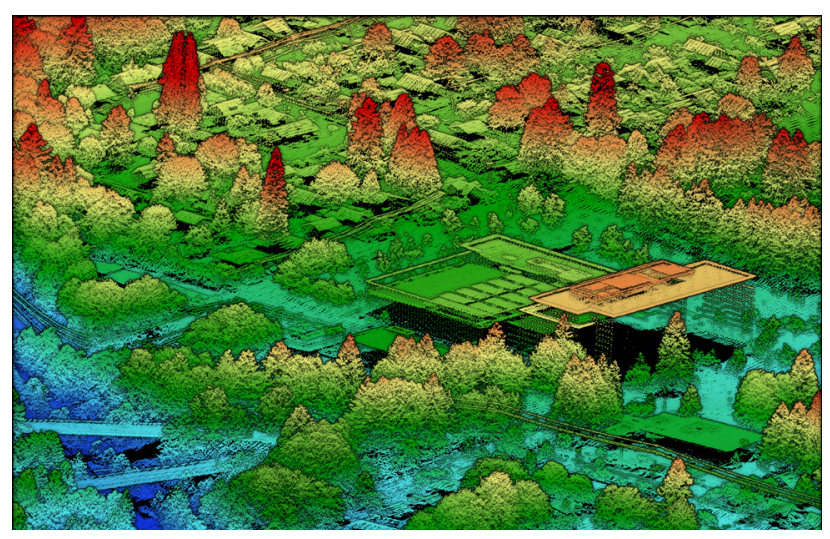

\section{Reference Cited}

Dewberry, 2012, Final report of the National Enhanced Elevation Assessment (revised March 29, 2012): Fairfax, Va., Dewberry, 84 p. plus appendixes, accessed July 6 , 2021, at https://www.dewberry.com/services/geospatial/national-enhanced-elevation-assessment.

Any use of trade, firm, or product names is for descriptive purposes only and does not imply endorsement by the U.S. Government.

\section{Learn More About 3DEP}

See the websites in the 3D Elevation Program sidebar and below. Please send questions to:

Director, National Geospatial Program

U.S. Geological Survey, MS 511

12201 Sunrise Valley Drive

Reston, VA 20192

Email: 3DEP@usgs.gov

$$
\text { -By Cindy A. Thatcher and Vicki Lukas }
$$

https://usgs.gov/3DEP

\section{D Elevation Program (3DEP)}

The 3D Elevation Program is managed by the U.S. Geological Survey (USGS) on behalf of the community of Federal, State, Tribal, local, and other partners and users of elevation data. In response to growing needs for high-quality elevation data, the goal of 3DEP is to complete acquisition of nationwide light detection and ranging (lidar) data (interferometric synthetic aperture radar [IfSAR] data in Alaska) to provide the first-ever national baseline of consistent, high-resolution topographic elevation data-both bare-earth digital elevation models and 3D point clouds.

\section{Benefits}

- Economies of scale by acquiring data for larger areas.

- Predictable and flexible Federal investments that reduce costs and allow better planning.

- Consistent national coverage that provides data for applications that span project, jurisdictional, and watershed boundaries.

- Simplified data acquisition that provides contracts, project management, quality assurance, and specifications for published data.

- National benefits of $\$ 690$ million per year conservatively, with the potential to generate $\$ 13$ billion per year in additional benefits through applications that span the economy (Dewberry, 2012).

\section{High-Quality Data and Products}

3DEP lidar provides coverage with a minimum of two points per square meter and a vertical error not to exceed 10 centimeters, measured as root mean square error (RMSE) in the vertical dimension. Lidar data products include all data points collected (point cloud) and a bare-earth digital elevation model with a 1-meter resolution. The USGS integrates the elevation data into The National Map. Data are available free of charge and without use restrictions. To download 3DEP products visit https://apps.nationalmap.gov/downloader/.

\section{Ways to Participate}

3DEP participation is open to Federal, State, Tribal, U.S. territorial, and local government partners, as well as private sector partners, and offers the option to acquire higher quality data. Partners may contribute funds toward projects managed by the USGS, or they may receive cooperative funds to manage their own projects. An annual Broad Agency Announcement is the mechanism used to establish partner agreements. Organizations and the private sector may contribute existing data that meet 3DEP requirements. For more information see the 3DEP website at https://usgs.gov/3DEP/collaborate. 